\title{
SINGULARITY FORMATION IN FRACTIONAL BURGERS' EQUATIONS
}

\author{
G. M. COCLITE, S. DIPIERRO, F. MADDALENA, AND E. VALDINOCI
}

\begin{abstract}
The formation of singularities in finite time in non-local Burgers' equations, with time-fractional derivative, is studied in detail. The occurrence of finite time singularity is proved, revealing the underlying mechanism, and precise estimates on the blow-up time are provided. The employment of the present equation to model a problem arising in job market is also analyzed.
\end{abstract}

\section{INTRODUCTION}

The study of singularities occurrence in nonlinear evolution problems constitutes a source of intriguing questions deeply related to the mathematical and physical issues. The basic example of a PDE evolution leading to shock formation is given by the so called Burgers' equation (actually introduced by Airy [1]) which represents a simple model for studying the interaction between nonlinear and dissipative phenomena. Moreover, this equation exhibits the basic nonlinear mechanism shared by the more involved nonlinearities inherent to Euler and Navier-Stokes equations [12]. In exploiting a possible scenario for singularity formation in nonlocal evolution problems, continuing a line of research pursued in [6] from a different perspective, here we investigate the effect of a nonlocal in time modification of Burgers' equation with respect to singularity creation.

Besides their interest from the purely mathematical point of view, the nonlocal operators with respect to the time variable find a number of concrete applications in many emerging fields of research like, for instance, the anomalous transportation problems (see [13]), the heat flow through ramified media (see [2]), and the theory of viscoelastic fluids (see Section 10.2 in [16] and the references therein). Specifically, we will also present here a concrete model from job market analysis

Date: June 3, 2019.

2010 Mathematics Subject Classification. 35L03, 35R11, 35L67, 35B44.

Key words and phrases. Finite time Blow-up. Anomalous transportation. Shock singularity.

The authors are members of the Gruppo Nazionale per l'Analisi Matematica, la Probabilità e le loro Applicazioni (GNAMPA) of the Istituto Nazionale di Alta Matematica (INdAM). SD and EV are supported by the Australian Research Council Discovery Project grant "Nonlocal Equations at Work" (NEW). SV is supported by the DECRA Project "Partial differential equations, free boundaries and applications". 
which naturally leads to a fractional Burgers' equation. See also Chapter 1 in [5] for several explicit motivations for fractional derivative problems.

Focusing on the case of inviscid fluid mechanics, we recall that in the classical Burgers' equation explicit examples show the possible formation of singularities in finite time, see [4]. In particular, an initial condition with unitary slope leads to a singularity at a unitary time.

The goal of this paper is to study whether a similar phenomenon persists in nonlocal Burgers' equations with a time-fractional derivative. That is, we investigate how a memory effect in the equation affects the singularity formation.

Our main results are the following:

- The memory effect does not prevent singularity formations.

- For initial data with unitary slopes, the blow-up time can be explicitly estimated from above, in a way that is uniform with respect to the memory effect (namely, it is not possible to slow down indefinitely the singularity formation using only memory effects).

- Explicit bounds from below of the blow-up times are also possible.

The precise mathematical setting in which we work is the following. First of all, to describe memory effects, we make use of the left-Caputo-derivative of order $\alpha \in$ $(0,1)$ with initial time $t_{0}$ for $t \in\left(t_{0},+\infty\right)$, defined by

$$
{ }^{C} D_{t_{0},+}^{\alpha} f(t):=\frac{1}{\Gamma(1-\alpha)} \int_{t_{0}}^{t} \frac{\dot{f}(\tau)}{(t-\tau)^{\alpha}} d \tau
$$

where $\Gamma$ is the Euler Gamma function.

In this framework, we consider the time-fractional Burgers' equation driven by the left-Caputo-derivative, given by

$$
\left\{\begin{array}{l}
{ }^{C} D_{0,+}^{\alpha} u(x, t)+u(x, t) \partial_{x} u(x, t)=0 \\
u(x, 0)=u_{0}(x)
\end{array} \quad \text { for all } x \in \mathbb{R} \text { and } t \in\left(0, T_{\star}\right),\right.
$$

In the recent literature, various types of fractional versions of the classical Burgers' equation were taken into account from different perspectives, see e.g. [15, 8, 3, 20, $7,17,21]$ and the references therein (in this paper, we also propose a simple motivation for equation (1.2) in Section 5). When $\alpha=1$, equation (1.2) reduces to the classical inviscid Burgers' equation

$$
\partial_{t} u(x, t)+u(x, t) \partial_{x} u(x, t)=0 .
$$

Remark 1.1. We notice that examples of solutions to classical Burgers' equation, exhibiting instantaneous and spontaneous formation of singularities, work well also in the present case. Indeed, the aim of our study relies in understanding, through quantitative estimates, how the finite-time creation of singularities can be affected by the presence of a fractional in time derivative. 
We prove that the time-fractional Burgers' equation driven by the left-Caputoderivative may develop singularities in finite time, according to the following result:

Theorem 1.2. There exist a time $T_{\star}>0$, a function $u_{0} \in C^{\infty}(\mathbb{R})$, a smooth solution $u: \mathbb{R} \times\left[0, T_{\star}\right) \rightarrow \mathbb{R}$ of the time-fractional Burgers' equation in (1.2) and a sequence $t_{n} \nearrow T_{\star}$ as $n \rightarrow+\infty$ such that

$$
\lim _{n \rightarrow+\infty} u\left(x, t_{n}\right)= \begin{cases}+\infty & \text { if } x \in(-\infty, 0), \\ -\infty & \text { if } x \in(0,+\infty) .\end{cases}
$$

Remark 1.3. The function $u$ in Theorem 1.2 will be constructed by taking

$$
u(x, t):=-x v(t),
$$

where $v$ is the solution of the time-fractional equation

$$
\left\{\begin{array}{l}
{ }^{C} D_{0,+}^{\alpha} v(t)=v^{2}(t) \quad \text { for } t \in\left(0, T_{\star}\right), \\
v(0)=1
\end{array}\right.
$$

When $\alpha=1$, the equation in (1.5) reduces to $\dot{v}(t)=v^{2}(t)$, which has the explicit solution $v(t)=1 /(1-t)$. Therefore, the function in (1.4) recovers the explicit, singular solution

$$
u(x, t)=-\frac{x}{1-t}
$$

of the classical Burgers' equation (1.3) as $\alpha \nearrow 1$. Of course, in the classical case, the blow-up time $T_{\star}$ of (1.6) is exactly 1: in this sense, our fractional construction in Theorem 1.2, recovers the classical case in the limit $\alpha \nearrow 1$.

We also observe that it is possible to give an explicit upper bound on the blow-up time for the fractional solution (1.4) in Theorem 1.2, as detailed in the following result:

Theorem 1.4. If $T_{\star}$ is the blow-up time found in Theorem 1.2, we have that

$$
T_{\star} \leqslant\left(\frac{1}{\Gamma(2-\alpha)}\right)^{1 / \alpha}
$$

In particular, for all $\alpha \in(0,1)$,

$$
T_{\star} \leqslant e^{1-\gamma}=1.52620511 \ldots,
$$

where $\gamma$ is the Euler-Mascheroni constant.

Remark 1.5. One can compare the general estimate in (1.8), valid for all $\alpha \in$ $(0,1)$, with the blow-up time for the classical solution in (1.6), in which $T_{\star}=1$. Indeed, we point out that the right hand side of (1.7) approaches 1 as $\alpha \nearrow$ 1. Hence, in view of Remark 1.3, we have that the bound in (1.7) is optimal when $\alpha \nearrow 1$. 
It is also possible to obtain a lower bound on the blow-up time involving the right hand side in (1.7), up to a reminder which is arbitrarily small as $\alpha \nearrow 1$. Indeed, we have the following result:

Theorem 1.6. If $T_{\star}$ is the blow-up time found in Theorem 1.2, we have that for any $\delta>0$ there exists $c_{\delta}>0$ such that

$$
T_{\star} \geqslant \frac{c_{\delta}^{\frac{1-\alpha}{\alpha}}}{1+\delta}\left(\frac{1}{\Gamma(2-\alpha)}\right)^{1 / \alpha} .
$$

Remark 1.7. We observe that the right hand side of (1.9) approaches $1 /(1+\delta)$ as $\alpha \nearrow 1$, which, for small $\delta$, recovers the unitary blow-up time of the classical solution in (1.6).

Remark 1.8. Of course, the blow-up time estimates in Theorems 1.4 and 1.6 are specific for the singular solution in (1.4), and other singular solutions have in general different blow-up times. As a matter of fact, by scaling, if $u$ is a solution of (1.2), then so is $u^{(\lambda)}(x, t):=u\left(\lambda^{\alpha} x, \lambda t\right)$, for all $\lambda>0$, with initial datum $u_{0}^{(\lambda)}(x):=u_{0}\left(\lambda^{\alpha} x\right)$. In particular, if $u$ is the function in (1.4) and $T_{\star}$ is its blow-up time, then the blow-up time of $u^{(\lambda)}$ is $T_{\star} / \lambda$. That is, when $\lambda \in(1,+\infty)$, the slope of the initial datum increases and accordingly the blow-up time becomes smaller. This is the reason for which we choose the setting in (1.4) to normalize the slope of the initial datum to be unitary.

Remark 1.9. It is interesting to observe the specific effect of the Caputo derivative on the solutions in simple and explicit examples. From our perspective, though the Caputo derivative is commonly viewed as a "memory" effect, the system does distinguish between a short-term memory effect, which enhances the role of the forcing terms, and a long-term memory effect, which is more keen to remember the past configurations.

To understand our point of view on this phenomenon, one can consider, for $\alpha \in$ $(0,1)$, the solution $u=u(t)$ of the linear equation

$$
\left\{\begin{array}{l}
{ }^{C} D_{0,+}^{\alpha} u(t)=\sum_{k=1}^{N} \delta_{p_{k}}(t), \\
u(0)=0,
\end{array}\right.
$$

where $0<p_{1}<\cdots<p_{N}$ and $\delta_{p}$ is the Dirac delta at the point $p \in \mathbb{R}$.

When $\alpha=1$, equation (1.10) reduces to the ordinary differential equation with impulsive forcing term given by

$$
\left\{\begin{array}{l}
\dot{u}(t)=\sum_{k=1}^{N} \delta_{p_{k}}(t), \\
u(0)=0 .
\end{array}\right.
$$


Up to negligible sets, the solution of (1.11) is the step function

$$
u(t)=\sharp\left\{k \in\{1, \ldots, N\} \text { s.t. } p_{k}<t\right\}=\sum_{\substack{1 \leqslant k \leqslant N \\ p_{k}<t}} 1 .
$$

On the other hand, equation (1.10) is a Volterra-type problem whose explicit solution is given by

$$
u(t)=\frac{1}{\Gamma(\alpha)} \sum_{\substack{1 \leqslant k \leqslant N \\ p_{k}<t}}\left(t-p_{k}\right)^{\alpha-1} .
$$

Notice that the solution in (1.13) recovers (1.12) as $\alpha \nearrow 1$. Nevertheless, the sharp geometric difference between the solutions in (1.12) and (1.13) is apparent (see Figure 1).

Indeed, while the classical solutions experience a unit jump at the times where the impulses take place, the structure of the fractional solutions exhibit a more complicated, and "less monotone", behavior. More specifically, on the one hand, for fractional solutions, the short-term memory effect of each impulse is to create a singularity towards infinity, and in this sense its impact on the solution is much stronger than in the classical case. On the other hand, the solution in (1.13) approaches zero outside the times in which the impulses occur, thus tending to recover the initial datum in view of a long-term memory effect.

The paper is organized as follows. Sections 2, 3, and 4 are devoted to the proofs of Theorems 1.2, 1.4, and 1.6, respectively. In Section 5 we propose a job market motivation for equation (1.2).

\section{Proof of Theorem 1.2}

The proof of Theorem 1.2 relies on a separation of variables method (as it will be apparent in the definition of the solution $u$ in (2.14) at the end of this proof). To make this method work, one needs a careful analysis of the solutions of timefractional equations, that we now discuss in details. Fixed $M \in \mathbb{N} \cap[4,+\infty)$, for any $r \in \mathbb{R}$ we define $f_{M}(r):=\min \left\{r^{2}, M^{2}\right\}$. We let $v_{M}$ be the solution of the Cauchy problem

$$
\left\{\begin{array}{l}
{ }^{C} D_{0,+}^{\alpha} v_{M}(t)=f_{M}\left(v_{M}(t)\right) \quad \text { for } t \in(0,+\infty) \\
v_{M}(0)=1
\end{array}\right.
$$

The existence and uniqueness of the solution $v_{M}$, which is continuous up to $t=0$, is warranted by Theorem 2 on page 304 of [9]. In addition, by Theorem 1 on page 300 of [9], we know that this solution can be represented in an integral form by the relation

$$
v_{M}(t)=1+\frac{1}{\Gamma(\alpha)} \int_{0}^{t} \frac{f_{M}\left(v_{M}(\tau)\right)}{(t-\tau)^{1-\alpha}} d \tau
$$



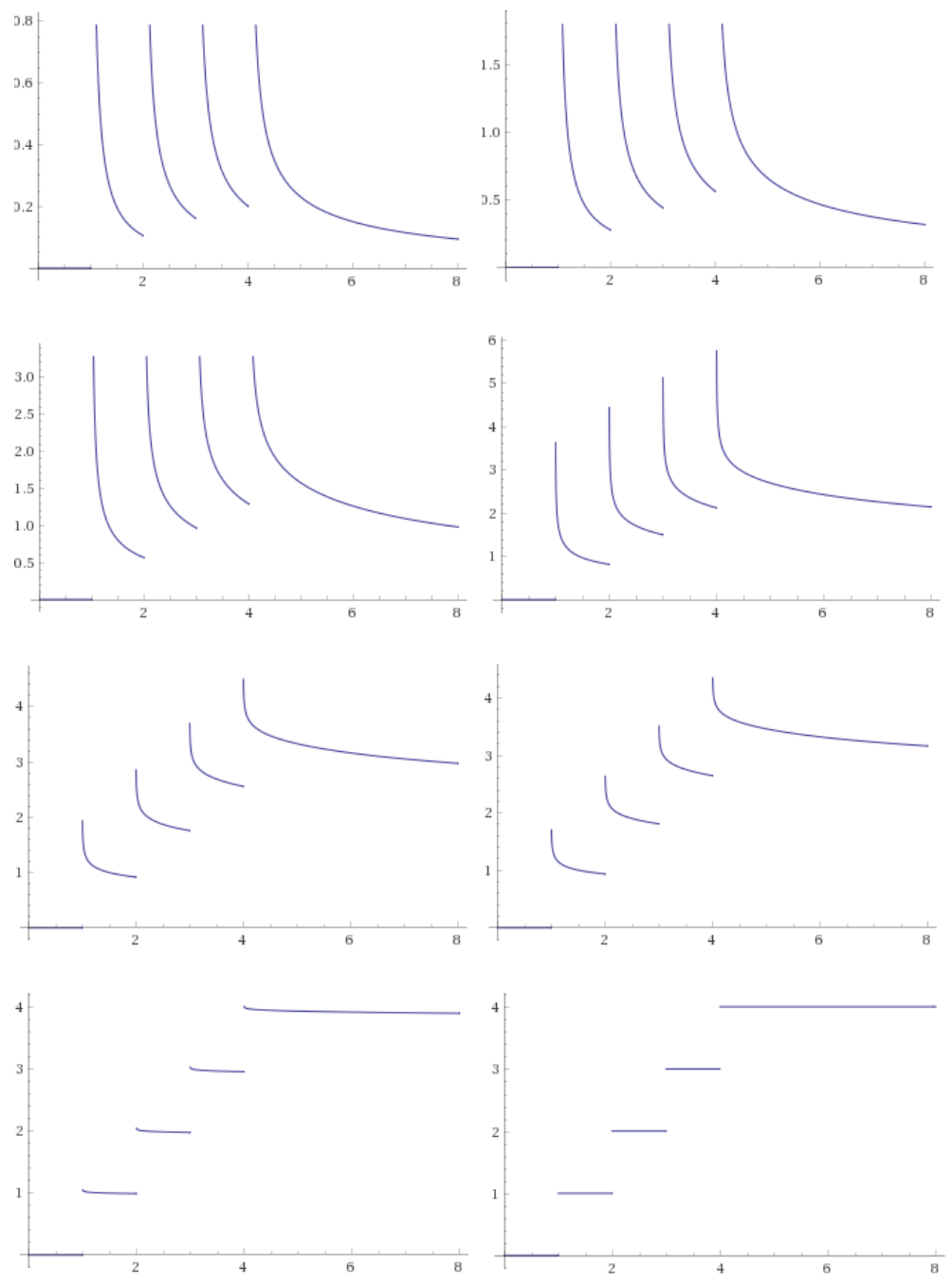

Figure 1. Plot of the solutions in (1.12) and (1.13) with the following parameters: $N=4, p_{1}=1, p_{2}=2, p_{3}=3$ and $p_{4}=4$. The different plots correspond to the cases $\alpha=\frac{1}{10}, \alpha=\frac{1}{4}, \alpha=\frac{1}{2}, \alpha=\frac{3}{4}$, $\alpha=\frac{7}{8}, \alpha=\frac{9}{10}, \alpha=\frac{99}{100}$, and $\alpha=1$.

In particular, since $f_{M} \geqslant 0$, we have that $v_{M} \geqslant 1$. Also, by continuity at $t=0$, there exists $\delta>0$ such that

$$
v_{4}(t) \leqslant 2 \text { for all } t \in(0, \delta) \text {. }
$$

We claim that

$$
v_{M}(t)=v_{4}(t) \text { for all } t \in(0, \delta) \text { and all } M \geqslant 4 \text {. }
$$


Indeed, if $t \in(0, \delta)$ and $M \geqslant 4$, we have that

$$
f_{M}\left(v_{4}(t)\right)=\min \left\{v_{4}^{2}(t), M^{2}\right\}=v_{4}^{2}(t)=\min \left\{v_{4}^{2}(t), 4^{2}\right\}=f_{4}\left(v_{4}(t)\right),
$$

thanks to (2.3), and therefore ${ }^{C} D_{0,+}^{\alpha} v_{4}(t)=f_{M}\left(v_{4}(t)\right)$ for all $t \in(0, \delta)$. Then, the uniqueness of the solution of the Cauchy problem in (2.1) gives (2.4), as desired.

Furthermore, we observe that if $M_{2} \geqslant M_{1}$ then $f_{M_{2}} \geqslant f_{M_{1}}$ and then

$$
{ }^{C} D_{0,+}^{\alpha} v_{M_{2}}(t)=f_{M_{2}}\left(v_{M_{2}}(t)\right) \geqslant f_{M_{1}}\left(v_{M_{2}}(t)\right) .
$$

Consequently, by the Comparison Principle ${ }^{1}$ in Theorem 4.10 on page 2894 in [14], we conclude that $v_{M_{2}} \geqslant v_{M_{1}}$. Therefore, for every $t \geqslant 0$, we can define

$$
v(t):=\lim _{M \rightarrow+\infty} v_{M}(t)=\sup _{M \in \mathbb{N} \cap[4,+\infty)} v_{M}(t) \in[1,+\infty) \cup\{+\infty\} .
$$

By (2.4), we know that

$$
v(t)=v_{4}(t) \leqslant \sup _{[0, \delta]} v_{4}<+\infty \quad \text { for all } t \in(0, \delta),
$$

and hence we can consider the largest $T_{\star} \in(0,+\infty) \cup\{+\infty\}$ such that

$$
\sup _{t \in\left[0, T_{0}\right]} v(t)<+\infty \quad \text { for all } T_{0} \in\left(0, T_{\star}\right) .
$$

By (2.6), we have that $T_{\star} \geqslant \delta$. We claim that

$$
\left\{\begin{array}{l}
{ }^{C} D_{0,+}^{\alpha} v(t)=v^{2}(t) \quad \text { for } t \in\left(0, T_{\star}\right), \\
v(0)=1
\end{array}\right.
$$

To prove this, we let $T_{0} \in\left(0, T_{\star}\right)$ and we exploit (2.7) to see that

$$
M_{0}:=\sup _{t \in\left[0, T_{0}\right]} v(t)<+\infty,
$$

and hence, for every $t \in\left(0, T_{0}\right)$ and every $M \geqslant M_{0}$,

$$
f_{M}\left(v_{M_{0}}(t)\right)=\min \left\{v_{M_{0}}^{2}(t), M^{2}\right\}=v_{M_{0}}^{2}(t)=\min \left\{v_{M_{0}}^{2}(t), M_{0}^{2}\right\}=f_{M_{0}}\left(v_{M_{0}}(t)\right) .
$$

This gives that ${ }^{C} D_{0,+}^{\alpha} v_{M_{0}}(t)=f_{M_{0}}\left(v_{M_{0}}(t)\right)=f_{M}\left(v_{M_{0}}(t)\right)$ for all $t \in\left(0, T_{0}\right)$ and $M \geqslant M_{0}$, and therefore, by the uniqueness of the solution of the Cauchy problem in (2.1), we find that $v_{M}=v_{M_{0}}$ in $\left(0, T_{0}\right)$. This and (2.5) give that

$$
M_{0} \geqslant v(t)=v_{M_{0}}(t) \quad \text { for all } t \in\left(0, T_{0}\right) .
$$

As a consequence, recalling (2.2), we obtain that, for all $t \in\left(0, T_{0}\right)$, the function $v$ satisfies the integral relation

$$
v(t)=v_{M_{0}}(t)=1+\frac{1}{\Gamma(\alpha)} \int_{0}^{t} \frac{f_{M_{0}}\left(v_{M_{0}}(\tau)\right)}{(t-\tau)^{1-\alpha}} d \tau
$$

\footnotetext{
${ }^{1}$ We observe that we cannot use here the Comparison Principle in Lemma 2.6 and Remark 2.1 on pages 219-220 in [19], since the monotonicity of the nonlinearity goes in the opposite direction.
} 


$$
=1+\frac{1}{\Gamma(\alpha)} \int_{0}^{t} \frac{f_{M_{0}}(v(\tau))}{(t-\tau)^{1-\alpha}} d \tau=1+\frac{1}{\Gamma(\alpha)} \int_{0}^{t} \frac{v^{2}(\tau)}{(t-\tau)^{1-\alpha}} d \tau
$$

and thus, by Theorem 1 in [9], we obtain (2.8), as desired.

Now we claim that

$$
T_{\star}<+\infty .
$$

To this end, we argue by contradiction and assume that $T_{\star}=+\infty$. We let $\lambda \geqslant 2$, $T>0$ (which will be taken as large as we wish in what follows), and

$$
\phi(t):= \begin{cases}\left(1-\frac{t}{T}\right)^{\lambda} & \text { if } t \in[0, T], \\ 0 & \text { if } t \in(T,+\infty) .\end{cases}
$$

We know (see Lemmata 1 and 2 in [10]) that

$$
\begin{aligned}
& \int_{0}^{T} D_{T,-}^{\alpha} \phi(t) d t=\frac{\lambda \Gamma(\lambda-\alpha)}{(\lambda-\alpha+1) \Gamma(\lambda-2 \alpha+1)} T^{1-\alpha} \\
& \int_{0}^{T} \frac{\left|D_{T,-}^{\alpha} \phi(t)\right|^{2}}{\phi(t)} d t=\frac{\lambda^{2}}{\lambda+1-2 \alpha}\left(\frac{\Gamma(\lambda-\alpha)}{\Gamma(\lambda+1-2 \alpha)}\right)^{2} T^{1-2 \alpha} .
\end{aligned}
$$

We also recall the left-Riemann-Liouville-derivative of order $\alpha \in(0,1)$ with initial time $t_{0}$ for $t \in\left(t_{0},+\infty\right)$, given by

$$
D_{t_{0},+}^{\alpha} f(t):=\frac{1}{\Gamma(1-\alpha)} \frac{d}{d t} \int_{t_{0}}^{t} \frac{f(\tau)}{(t-\tau)^{\alpha}} d \tau,
$$

and we point out that

$$
{ }^{C} D_{t_{0},+}^{\alpha} f(t)=D_{t_{0},+}^{\alpha}\left(f(t)-f\left(t_{0}\right)\right) .
$$

This and (2.8) give that

$$
v^{2}(t)={ }^{C} D_{0,+}^{\alpha} v(t)=D_{0,+}^{\alpha}(v(t)-v(0))=D_{0,+}^{\alpha} w(t),
$$

where $w(t):=v(t)-1$.

It is also useful to consider the right-Riemann-Liouville-derivative of order $\alpha \in$ $(0,1)$ with final time $t_{0}$ for $t \in\left(-\infty, t_{0}\right)$, given by

$$
D_{t_{0},-}^{\alpha} f(t):=-\frac{1}{\Gamma(1-\alpha)} \frac{d}{d t} \int_{t}^{t_{0}} \frac{f(\tau)}{(\tau-t)^{\alpha}} d \tau
$$

Integrating by parts (see Corollary 2 on page 46 of [18], or formula (15) in [11]), and recalling (2.11), we obtain that

$$
\begin{aligned}
\int_{0}^{T} \phi(t) v^{2}(t) d t & =\int_{0}^{T} \phi(t) D_{0,+}^{\alpha} w(t) d t \\
& =\int_{0}^{T} D_{T,-}^{\alpha} \phi(t) w(t) d t=\int_{0}^{T} D_{T,-}^{\alpha} \phi(t)(v(t)-1) d t .
\end{aligned}
$$


From this and (2.10) we find that

$$
\int_{0}^{T} \phi(t) v^{2}(t) d t=\int_{0}^{T} D_{T,-}^{\alpha} \phi(t) v(t) d t-C_{1} T^{1-\alpha}
$$

for some $C_{1}>0$ independent of $T$.

Furthermore,

$$
\begin{aligned}
\int_{0}^{T} D_{T,-}^{\alpha} \phi(t) v(t) d t & =\int_{0}^{T} \frac{D_{T,-}^{\alpha} \phi(t)}{\sqrt{\phi(t)}} \sqrt{\phi(t)} v(t) d t \\
& \leqslant \frac{1}{2} \int_{0}^{T} \frac{\left|D_{T,-}^{\alpha} \phi(t)\right|^{2}}{\phi(t)} d t+\frac{1}{2} \int_{0}^{T} \phi(t) v^{2}(t) d t \\
& =C_{2} T^{1-2 \alpha}+\frac{1}{2} \int_{0}^{T} \phi(t) v^{2}(t) d t
\end{aligned}
$$

thanks to (2.10), for some $C_{2}>0$ independent of $T$. As a consequence, recalling (2.12), we conclude that

$$
\frac{1}{2} \int_{0}^{T} \phi(t) v^{2}(t) d t \leqslant C_{2} T^{1-2 \alpha}-C_{1} T^{1-\alpha} .
$$

Therefore, recalling that $v \geqslant 1$ in view of $(2.5)$,

$$
C_{2} T^{1-2 \alpha}-C_{1} T^{1-\alpha} \geqslant \frac{1}{2} \int_{0}^{T} \phi(t) d t=\frac{1}{2} \int_{0}^{T}\left(1-\frac{t}{T}\right)^{\lambda} d t=\frac{T}{2(1+\lambda)},
$$

and accordingly

$$
0=\lim _{T \rightarrow+\infty} C_{2} T^{-2 \alpha}-C_{1} T^{-\alpha} \geqslant \frac{1}{2(1+\lambda)},
$$

which is a contradiction, thus completing the proof of (2.9).

Then, from (2.7) and (2.9), we obtain that

$$
\limsup _{t \nearrow T_{\star}} v(t)=+\infty .
$$

Hence, we consider a sequence $t_{n} \nearrow T_{\star}$ such that

$$
\lim _{n \rightarrow+\infty} v\left(t_{n}\right)=+\infty
$$

and we define

$$
u(x, t):=-x v(t) .
$$

For every $t \in\left(0, T_{\star}\right)$, we have that

$$
{ }^{C} D_{0,+}^{\alpha} u(x, t)+u(x, t) \partial_{x} u(x, t)=-x^{C} D_{0,+}^{\alpha} v(t)+x v^{2}(t)=0,
$$

thanks to $(2.8)$, and also $u(x, 0)=-x v(0)=-x$. These observations and (2.13) prove Theorem 1.2. 


\section{Proof of Theorem 1.4}

We set

$$
b=b(\alpha):=\left(\frac{1}{\Gamma(2-\alpha)}\right)^{1 / \alpha}
$$

For any $t \in(0, b)$, let also

$$
w(t):=\frac{b}{b-t} .
$$

Notice that $w(0)=1$. Moreover, for any $t \in(0, b)$ and any $\tau \in(0, t)$, we have that

$$
\dot{w}(\tau)=\frac{b}{(b-\tau)^{2}} \leqslant \frac{b}{(b-t)^{2}}=\frac{w^{2}(t)}{b} .
$$

Consequently, by (1.1), for all $t \in(0, b)$,

$$
\begin{aligned}
{ }^{C} D_{0,+}^{\alpha} w(t) & =\frac{1}{\Gamma(1-\alpha)} \int_{0}^{t} \frac{\dot{w}(\tau)}{(t-\tau)^{\alpha}} d \tau \leqslant \frac{w^{2}(t)}{b \Gamma(1-\alpha)} \int_{0}^{t} \frac{d \tau}{(t-\tau)^{\alpha}} \\
& =\frac{t^{1-\alpha} w^{2}(t)}{b \Gamma(1-\alpha)(1-\alpha)}=\frac{t^{1-\alpha} w^{2}(t)}{b \Gamma(2-\alpha)} \leqslant \frac{b^{1-\alpha} w^{2}(t)}{b \Gamma(2-\alpha)} \\
& =\frac{w^{2}(t)}{b^{\alpha} \Gamma(2-\alpha)}=w^{2}(t) .
\end{aligned}
$$

Therefore, using the Comparison Principle in Theorem 4.10 on page 2894 in [14], if $v$ is as in (2.8), we find that $v \geqslant w$ in their common domain of definition. This, (2.14), and the fact that $w$ diverges at $t=b$ yield that

$$
T_{\star} \leqslant b=b(\alpha),
$$

which, together with (3.1), establishes (1.7), as desired.

Now we prove (1.8). For this, we first show that the map $(0,1) \ni \alpha \mapsto b(\alpha)$ that was introduced in (3.1) is monotone. To this end, we recall the polygamma functions for $\tau \in(1,2)$ and $n \in \mathbb{N}$ with their integral representations, namely

$$
\begin{aligned}
& \psi_{n}(\tau):=\left(\frac{d}{d \tau}\right)^{n+1} \log (\Gamma(\tau)) \quad \text { for all } n \in\{0,1,2,3, \ldots\}, \\
& (-1)^{n+1} \int_{0}^{\infty} \frac{t^{n} e^{-t \tau}}{1-e^{-t}} d t \quad \text { for all } n \in\{1,2,3, \ldots\} .
\end{aligned}
$$

We observe, in particular, that, for all $\tau \in(1,2)$,

$$
\psi_{1}(\tau)=\int_{0}^{\infty} \frac{t e^{-t \tau}}{1-e^{-t}} d t>0 .
$$

Let also, for all $\tau \in(1,2)$,

$$
\xi(\tau):=\log (\Gamma(\tau))+(2-\tau) \psi_{0}(\tau) .
$$


We see that

$$
\xi^{\prime}(\tau)=\psi_{0}(\tau)-\psi_{0}(\tau)+(2-\tau) \psi_{1}(\tau)>0
$$

thanks to (3.3) and therefore, for all $\tau \in(1,2)$,

$$
0<\int_{\tau}^{2} \xi^{\prime}(\sigma) d \sigma=\xi(2)-\xi(\tau)=\log (\Gamma(2))-\xi(\tau)=-\xi(\tau) .
$$

Now we define

$$
\lambda(\tau):=\frac{\log (\Gamma(\tau))}{2-\tau} .
$$

We have that, for all $\tau \in(1,2)$,

$$
\lambda^{\prime}(\tau)=\frac{\log (\Gamma(\tau))}{(2-\tau)^{2}}+\frac{\psi_{0}(\tau)}{2-\tau}=\frac{\xi(\tau)}{(2-\tau)^{2}}<0,
$$

due to (3.4).

Therefore, the function $(1,2) \ni \tau \mapsto \lambda(\tau)$ is decreasing, and hence so is the function $(1,2) \ni \tau \mapsto e^{\lambda(\tau)}=: \Lambda(\tau)$. Hence, using the substitution $\tau:=2-\alpha$, with $\alpha \in(0,1)$, we deduce that the following function is increasing:

$$
\begin{aligned}
\Lambda(2-\alpha) & =e^{\lambda(2-\alpha)}=\exp \left(\frac{\log (\Gamma(2-\alpha))}{\alpha}\right) \\
& =\exp \left(\log \left(\Gamma^{1 / \alpha}(2-\alpha)\right)\right)=\Gamma^{1 / \alpha}(2-\alpha)=\frac{1}{b(\alpha)},
\end{aligned}
$$

thanks to (3.1).

Consequently, the function $(0,1) \ni \alpha \mapsto b(\alpha)$ is decreasing, hence it attains its maximum as $\alpha \searrow 0$. This and (3.2) give that

$$
T_{\star} \leqslant \lim _{\alpha \searrow 0} b(\alpha) .
$$

Furthermore, using L'Hôpital's Rule,

$$
\lim _{\alpha \searrow 0} \frac{\log (\Gamma(2-\alpha))}{\alpha}=-\lim _{\alpha \searrow 0} \psi_{0}(2-\alpha)=-\psi_{0}(2)=\gamma-1,
$$

where $\gamma$ is the Euler-Mascheroni constant, and therefore

$$
\lim _{\alpha \searrow 0} b(\alpha)=\lim _{\alpha \searrow 0} \exp \left(-\frac{\log (\Gamma(2-\alpha))}{\alpha}\right)=e^{1-\gamma} .
$$

This and (3.5) give the desired result in (1.8). 


\section{Proof of Theorem 1.6}

We let $\delta>0$ as in the statement of Theorem 1.6, and

$$
\kappa:=\sqrt{1+\delta}-1>0 .
$$

We define

$$
\begin{aligned}
\eta:=\frac{(1+\kappa)^{2}}{\kappa^{2}}, & d:=\left(\frac{1}{\Gamma(2-\alpha) \kappa \eta(1+\eta)}\right)^{\frac{1}{\alpha}}, \\
a:=\frac{\Gamma(2-\alpha)}{d^{1-\alpha}}, & b:=(1+\kappa) a .
\end{aligned}
$$

Let also

$$
T:=\frac{1}{b}-(1+\eta) d
$$

In light of (4.2), we remark that

$$
\begin{aligned}
T & =\frac{1}{(1+\kappa) a}-(1+\eta) d \\
& =\frac{d^{1-\alpha}}{(1+\kappa) \Gamma(2-\alpha)}-(1+\eta) d \\
& =(1+\eta) d\left(\frac{d^{-\alpha}}{(1+\kappa) \Gamma(2-\alpha)(1+\eta)}-1\right) \\
& =(1+\eta) d\left(\frac{\kappa \eta}{1+\kappa}-1\right) \\
& =(1+\eta) d\left(\frac{1+\kappa}{\kappa}-1\right) \\
& =\frac{(1+\eta) d}{\kappa} \\
& =\frac{1+\eta}{\kappa}\left(\frac{1}{\Gamma(2-\alpha) \kappa \eta(1+\eta)}\right)^{\frac{1}{\alpha}} \\
& =\frac{1}{(\Gamma(2-\alpha))^{\frac{1}{\alpha}} \kappa^{\frac{1+\alpha}{\alpha}} \eta^{\frac{1}{\alpha}}(1+\eta)^{\frac{1-\alpha}{\alpha}}} \\
& =\frac{\kappa^{\frac{3(1-\alpha)}{\alpha}}}{(\Gamma(2-\alpha))^{\frac{1}{\alpha}}(1+\kappa)^{\frac{2}{\alpha}}\left(1+2 \kappa+2 \kappa^{2}\right)^{\frac{1-\alpha}{\alpha}}} .
\end{aligned}
$$

Recalling (4.1), we can also define

$$
c_{\delta}:=\frac{\kappa^{3}}{(1+\kappa)^{2}\left(1+2 \kappa+2 \kappa^{2}\right)},
$$


and then (4.3) becomes

$$
T=\frac{c_{\delta}^{\frac{1-\alpha}{\alpha}}}{(\Gamma(2-\alpha))^{\frac{1}{\alpha}}(1+\kappa)^{2}}=\frac{c_{\delta}^{\frac{1-\alpha}{\alpha}}}{(\Gamma(2-\alpha))^{\frac{1}{\alpha}}(1+\delta)},
$$

which coincides with the right hand side of (1.9).

Therefore, to complete the proof of Theorem 1.6, it is enough to show that

$$
T_{\star} \geqslant T \text {. }
$$

To this end, for all $t \in(0, T)$, we define

$$
z(t):=\frac{b}{a(1-b t)}+1-\frac{b}{a} .
$$

Notice that $z(0)=1$. Moreover, for all $t \in(0, T)$ and $\tau \in(0, t)$ we have that

$$
\begin{aligned}
\frac{b}{a(1-b \tau)}-z(\tau+d) & =\frac{b}{a(1-b \tau)}-\frac{b}{a(1-b d-b \tau)}-1+\frac{b}{a} \\
& =\frac{1+\kappa}{1-b \tau}-\frac{1+\kappa}{1-b d-b \tau}+\kappa \\
& =-\frac{(1+\kappa) b d}{(1-b \tau)(1-b d-b \tau)}+\kappa \\
& \geqslant-\frac{(1+\kappa) b d}{(1-b T)(1-b d-b T)}+\kappa \\
& =-\frac{(1+\kappa)}{(1+\eta) \eta b d}+\kappa
\end{aligned}
$$

Hence, since

$$
b d=(1+\kappa) a d=(1+\kappa) \Gamma(2-\alpha) d^{\alpha}=\frac{(1+\kappa)}{\kappa \eta(1+\eta)},
$$

we see from (4.6) that

$$
\frac{b}{a(1-b \tau)}-z(\tau+d) \geqslant-\kappa+\kappa=0
$$

and therefore

$$
\frac{b^{2}}{a^{2}(1-b \tau)^{2}} \geqslant z^{2}(\tau+d) .
$$

As a consequence, we conclude that

$$
\dot{z}(\tau)=\frac{b^{2}}{a(1-b \tau)^{2}} \geqslant a z^{2}(\tau+d) .
$$

Accordingly, by (1.1), for all $t \in(0, T)$,

$$
{ }^{C} D_{0,+}^{\alpha} z(t)=\frac{1}{\Gamma(1-\alpha)} \int_{0}^{t} \frac{\dot{z}(\tau)}{(t-\tau)^{\alpha}} d \tau
$$




$$
\geqslant \frac{a}{\Gamma(1-\alpha)} \int_{t-d}^{t} \frac{z^{2}(\tau+d)}{(t-\tau)^{\alpha}} d \tau
$$

Consequently, using the fact that $z$ is increasing,

$$
{ }^{C} D_{0,+}^{\alpha} z(t) \geqslant \frac{a}{\Gamma(1-\alpha)} \int_{t-d}^{t} \frac{z^{2}(t)}{(t-\tau)^{\alpha}} d \tau=\frac{a d^{1-\alpha} z^{2}(t)}{\Gamma(2-\alpha)}=z^{2}(t) .
$$

Then, recalling (2.8) and exploiting the Comparison Principle in Theorem 4.10 on page 2894 in [14], we obtain that $v \leqslant z$ in their common domain of definition. In particular, this gives (4.5), and so the proof of Theorem 1.6 is complete.

\section{A motivation for (1.2) From the Job MARKet}

In this section we give a simple, but concrete, motivation for the time-fractional Burgers' equation in (1.2) making a model of an ideal job market from a few basic principles. The discussion that we present here is a modification of classical models proposed for fluid dynamics and traffic flow in a highway.

We fix parameters $\delta, \varepsilon>0$ and we use the real line to describe the positions available in a company, in which workers can decide to work. More specifically, the working levels in the company are denoted by $x \in \varepsilon \mathbb{Z}$ and the higher the value of $x$ the higher and more appealing the position is (e.g., $x=\varepsilon$ corresponds to Brigadier, $x=2 \varepsilon$ to Major, $x=3 \varepsilon$ to Lieutenant, $x=4 \varepsilon$ to General, etc.).

We suppose that the main motivation for a worker to join the company by taking the position $x \in \varepsilon \mathbb{Z}$ at time $t \in \delta \mathbb{N}$ is provided by the possibility of career progression towards the successive level. If we denote by $\rho$ the number of people employed in a given position at a given time, and by $v$ the velocity of career progression relative to a given position at a given time, the "group velocity" of career progression for a given position at a given time is obtained by the product $p:=\rho v$.

We suppose that the potential worker who is possibly entering the company at the level $x \in \varepsilon \mathbb{Z}$ will look at the value of $p$ for its perspective position and compare it with the value of $p$ relative to subsequent level $x+\varepsilon$, and this will constitute, in this model, the main drive for the worker to join the company. At time $t \in \delta \mathbb{N}$, this driving force is therefore quantified by

$$
\mathcal{D}(x, t):=\hat{c}(p(x+\varepsilon, t)-p(x, t))=\hat{c}(\rho(x+\varepsilon, t) v(x+\varepsilon, t)-\rho(x, t) v(x, t)),
$$

for a normalizing constant $\hat{c}>0$ Then, we assume that the potential worker base her or his decision not only considering the driving force at the present time, but also taking into account the past history of the company. Past events will be weighted by a kernel $\mathcal{K}$, to make the information coming from remote times less important than the ones relative to the contemporary situation. For concreteness, we suppose that the information coming from the time $t-\tau$, with $t=\delta N, N \in \mathbb{N}$, 
and $\tau \in\{\delta, 2 \delta, \ldots, \delta N\}$, is weighted by the kernel

$$
\mathcal{K}(\tau):=\frac{\delta}{\tau^{\beta}}, \quad \text { for some } \beta \in(0,1)
$$

If all the potential workers argue in this way, the number of workers at time $t=\delta N$ in the working position $x \in \varepsilon \mathbb{Z}$ of the company is given by the initial number of workers, incremented by the effect of the drive function in the history of the company, according to the memory effect that we have described, that is

$$
\rho(x, t)=\rho(x, \delta N)=\rho(x, 0)+c \sum_{j=1}^{N} \mathcal{D}(x, t-\delta j) \mathcal{K}(\delta j),
$$

for some normalizing constant $c>0$. Hence, exploiting (5.2),

$$
\rho(x, t)=\rho(x, 0)+c \sum_{j=1}^{N} \mathcal{D}(x, t-\delta j) \frac{\delta}{(\delta j)^{\beta}} .
$$

Using the Riemann sum approximation of an integral, for small $\delta$ we can substitute the summation in the right hand side of (5.3) with an integral, and, with this asymptotic procedure, we replace (5.3) with

$$
\rho(x, t)=\rho(x, 0)+c \int_{0}^{t} \mathcal{D}(x, t-\tau) \frac{d \tau}{\tau^{\beta}} .
$$

Then, we define $\alpha:=1-\beta \in(0,1)$ and, up to a time scale, we choose $c:=1 / \Gamma(\alpha)$. In this way, we can write (5.4) as

$$
\begin{aligned}
\rho(x, t) & =\rho(x, 0)+\frac{1}{\Gamma(\alpha)} \int_{0}^{t} \mathcal{D}(x, t-\tau) \frac{d \tau}{\tau^{1-\alpha}} \\
& =\rho(x, 0)+\frac{1}{\Gamma(\alpha)} \int_{0}^{t} \mathcal{D}(x, \sigma) \frac{d \sigma}{(t-\sigma)^{1-\alpha}},
\end{aligned}
$$

or, equivalently (see e.g. Theorem 1 on page 300 of [9]),

$$
{ }^{C} D_{0,+}^{\alpha} \rho(x, t)=\mathcal{D}(x, t) .
$$

Thus, recalling (5.1) (and using the normalization $\hat{c}:=1 / \varepsilon$ ),

$$
{ }^{C} D_{0,+}^{\alpha} \rho(x, t)=\frac{\rho(x+\varepsilon, t) v(x+\varepsilon, t)-\rho(x, t) v(x, t)}{\varepsilon},
$$

and then, in the approximation of $\varepsilon$ small,

$$
{ }^{C} D_{0,+}^{\alpha} \rho(x, t)=\partial_{x}(\rho(x, t) v(x, t)) .
$$

Now we make the ansatz that the career velocity is mainly influenced by the number of people in a given position, namely this velocity is proportional to the 
"vacancies" in a given working level. If $\rho_{\max } \in(0,+\infty)$ is the maximal number of workers that the market allows in any given position, we therefore assume that

$$
v=\tilde{c}\left(\rho_{\max }-\rho\right),
$$

for a normalizing constant $\tilde{c}>0$. Of course, in more complicated models, one can allow $\rho_{\max }$ and $\tilde{c}$ to vary in space and time, but we will take them to be constant to address the simplest possible case, and in fact, for simplicity, up to scalings, we take $\rho_{\max }=1$ and $\tilde{c}=1$.

Then, plugging (5.6) into (5.5), we obtain

$$
{ }^{C} D_{0,+}^{\alpha} \rho(x, t)=\partial_{x}(\rho(x, t)(1-\rho(x, t))) .
$$

Now we perform the substitution

$$
u(x, t):=2 \rho(x, t)-1,
$$

and we thereby conclude that

$$
\begin{aligned}
{ }^{C} D_{0,+}^{\alpha} u(x, t) & =2^{C} D_{0,+}^{\alpha} \rho(x, t) \\
& =2 \partial_{x}(\rho(x, t)(1-\rho(x, t))) \\
& =2 \partial_{x}\left(\frac{u(x, t)+1}{2}\left(1-\frac{u(x, t)+1}{2}\right)\right) \\
& =2 \partial_{x}\left(\frac{u(x, t)+1}{2}-\frac{u^{2}(x, t)+2 u(x, t)+1}{4}\right) \\
& =2 \partial_{x}\left(\frac{1}{4}-\frac{u^{2}(x, t)}{4}\right) \\
& =-u(x, t) \partial_{x} u(x, t),
\end{aligned}
$$

which corresponds to (1.2).

We remark that in the model above one can interpret $\rho \in \mathbb{R}$ also when it takes negative values, e.g. as a position vacancy. As a matter of fact, since the driving force of equation (5.7) can be written as $\partial_{x} \rho(1-2 \rho)$, we observe that such a drive becomes "stronger" for negative values of $\rho$ (that is, vacancies in the job markets tend to increase the number of filled positions).

It is also interesting to interpret the result in Theorem 1.2 in the light of the motivation discussed here and recalling the setting in (5.8). Indeed, the value $1 / 2$ for the working force $\rho$ plays a special role in our framework since not only it corresponds to the average between the null working force and the maximal one allowed by the market, but also, and most importantly, to the critical value of the concave function $\rho(1-\rho)$, whose derivative is the driving force of equation (5.7). 
In this spirit, recalling (1.4), we have that the solution found in Theorem 1.2 takes the form

$$
\rho(x, t)=\frac{1-x v(t)}{2},
$$

for a function $v$ which is diverging in finite time. The expression in (5.9) says that the role corresponding to the job position $x=0$ has, at the initial time, exactly the critical working force $\rho=1 / 2$. Given the linear structure in $x$ of the solution in (5.9), this says that the job position corresponding to $x=0$ will maintain its critical value $\rho=1 / 2$ for all times, while higher level job roles will experience a dramatic loss of number of positions available (and, correspondingly, lower level job roles a dramatic increase). Though it is of course unrealistic that the job market really attains an (either positive or negative) infinite value in a finite time, and the model presented in equation (5.7) must necessarily "break" for too large values of $\rho$ (which, of course, in practice, cannot exceed the total working population), we think that solutions such as (5.9) may represent a concrete case in which the market would in principle allow arbitrarily high level job positions, but in practice (almost) all the workers end up obtaining a position level below a certain threshold (in this case normalized to $x=0$ ), which constitutes a "de facto" optimal role allowed by the evolution of special preexisting conditions.

\section{REFERENCES}

[1] G. Airy. Tides and Waves: Extracted from the Encyclopaedia Metropolitana, Tom. V Pag. 241 - 396. William Clowes and Sons, 1845.

[2] V. E. Arkhincheev and E. Baskin. Anomalous diffusion and drift in a comb model of percolation clusters. Journal of Experimental and Theoretical Physics, 73:161-165, 1991.

[3] P. Artale Harris and R. Garra. Analytic solution of nonlinear fractional Burgers-type equation by invariant subspace method. Nonlinear Studies, 20:471-481, 062013.

[4] A. Bressan. Hyperbolic systems of conservation laws, volume 20 of Oxford Lecture Series in Mathematics and its Applications. Oxford University Press, Oxford, 2000. The onedimensional Cauchy problem.

[5] A. Carbotti, S. Dipierro, and E. Valdinoci. Local density of solutions to fractional equations. De Gruyter Studies in Mathematics, De Gruyter, Berlin, 2019.

[6] G. M. Coclite, S. Dipierro, F. Maddalena, and E. Valdinoci. Wellposedness of a nonlinear peridynamic model. Nonlinearity, 32(1):1-21, nov 2018.

[7] A. Esen and O. Tasbozan. Numerical solution of time fractional Burgers equation by cubic b-spline finite elements. Mediterranean Journal of Mathematics, 13(3):1325-1337, Jun 2016.

[8] M. Inc. The approximate and exact solutions of the space- and time-fractional Burgers equations with initial conditions by variational iteration method. Journal of Mathematical Analysis and Applications, 345(1):476 - 484, 2008.

[9] A. Kilbas and S. Marzan. Cauchy problem for differential equation with Caputo derivative. Fractional Calculus and Applied Analysis, 7(3):297-321, 2004.

[10] M. Kirane and K. Furati. Necessary Conditions for the Existence of Global Solutions to Systems of Fractional Differential Equations. Fractional Calculus and Applied Analysis, 11(3):281-298, 2008. 
[11] M. Kirane and S. A. Malik. The profile of blowing-up solutions to a nonlinear system of fractional differential equations. Nonlinear Analysis: Theory, Methods 8 Applications, $73(12): 3723-3736,2010$.

[12] A. Kiselev and V. Šverák. Small scale creation for solutions of the incompressible twodimensional Euler equation. Ann. of Math. (2), 180(3):1205-1220, 2014.

[13] B. Li and J. Wang. Anomalous heat conduction and anomalous diffusion in one-dimensional systems. Physical Review Letters, 91:044301, Jul 2003.

[14] L. Li and J. Liu. A generalized definition of Caputo derivatives and its application to fractional ODEs. SIAM Journal on Mathematical Analysis, 50(3):2867-2900, 2018.

[15] P. Miškinis. Some properties of fractional burgers equation. Mathematical Modelling and Analysis, 7(1):151-158, 2002.

[16] I. Podlubny. Fractional differential equations, volume 198 of Mathematics in Science and Engineering. Academic Press, Inc., San Diego, CA, 1999. An introduction to fractional derivatives, fractional differential equations, to methods of their solution and some of their applications.

[17] K. M. Saad and E. H. F. Al-Sharif. Analytical study for time and time-space fractional Burgers' equation. Advances in Difference Equations, 2017(1):300, Sep 2017.

[18] S. Samko, A. Kilbas, and O. Marichev. Fractional Integrals and Derivatives. Taylor \& Francis, 1993.

[19] V. Vergara and R. Zacher. Optimal decay estimates for time-fractional and other nonlocal subdiffusion equations via energy methods. SIAM Journal on Mathematical Analysis, 47(1):210-239, 2015.

[20] G.-C. Wu and D. Baleanu. Variational iteration method for the Burgers' flow with fractional derivatives - new Lagrange multipliers. Applied Mathematical Modelling, 37(9):6183 - 6190, 2013.

[21] A. f. Yokuş and D. Kaya. Numerical and exact solutions for time fractional Burgers' equation. Journal of Nonlinear Sciences and Applications. JNSA, 10(7):3419-3428, 2017.

(Giuseppe Maria Coclite and Francesco Maddalena)

Department of Mechanics, Mathematics and Management, Polytechnic of Bari, Via E. Orabona 4, 70125 Bari, Italy.

E-mail address: giuseppemaria.coclite@poliba.it, francesco.maddalena@poliba.it

(Serena Dipierro and Enrico Valdinoci)

Department of Mathematics and Statistics, University of Western Australia, 35

Stirling Highway, Crawley, WA 6009, Australia.

E-mail address: serena.dipierro@uwa.edu.au, enrico.valdinoci@uwa.edu.au 